\title{
An Exploratory Descriptive Study of Antimicrobial Resistance Patterns of Staphylococcus Spp. Isolated from Horses Presented at a Veterinary Teaching Hospital
}

\author{
James Wabwire Oguttu', Daniel Nenene Qekwana ${ }^{2}$ and Agricola Odoi ${ }^{1,3^{*}}$ (D)
}

\begin{abstract}
Background: Antimicrobial resistant Staphylococcus are becoming increasingly important in horses because of the zoonotic nature of the pathogens and the associated risks to caregivers and owners. Knowledge of the burden and their antimicrobial resistance patterns are important to inform control strategies. This study is an exploratory descriptive investigation of the burden and antimicrobial drug resistance patterns of Staphylococcus isolates from horses presented at a veterinary teaching hospital in South Africa.
\end{abstract}

Methods: Retrospective laboratory clinical records of 1027 horses presented at the University of Pretoria veterinary teaching hospital between 2007 and 2012 were included in the study. Crude and factor-specific percentages of Staphylococcus positive samples, antimicrobial resistant (AMR) and multidrug resistant (MDR) isolates were computed and compared across Staphylococcus spp., geographic locations, seasons, years, breed and sex using Chi-square and Fisher's exact tests.

Results: Of the 1027 processed clinical samples, 12.0\% were Staphylococcus positive. The majority of the isolates were S. aureus (41.5\%) followed by S. pseudintermedius (14.6\%). Fifty-two percent of the Staphylococcus positive isolates were AMR while $28.5 \%$ were MDR. Significant $(p<0.05)$ differences in the percentage of samples with isolates that were AMR or MDR was observed across seasons, horse breeds and Staphylococcus spp. Summer season had the highest (64.3\%) and autumn the lowest (29.6\%) percentages of AMR isolates. Highest percentage of AMR samples were observed among the Boerperds (85.7\%) followed by the American saddler (75\%) and the European warm blood (73.9\%). Significantly $(p<0.001)$ more S. aureus isolates (72.5\%) were AMR than S. pseudintermedius isolates (38.9\%). Similarly, significantly $(p<0.001)$ more $S$. aureus (52.9\%) exhibited MDR than S. pseudintermedius (16.7\%). The highest levels of AMR were towards $\beta$-lactams (84.5\%) followed by trimethoprim/sulfamethoxazole (folate pathway inhibitors) (60.9\%) while the lowest levels of resistance were towards amikacin (14.\%).

Conclusions: This exploratory study provides useful information to guide future studies that will be critical for guiding treatment decisions and control efforts. There is a need to implement appropriate infection control, and judicious use of antimicrobials to arrest development of antimicrobial resistance. A better understanding of the status of the problem is a first step towards that goal.

Keywords: Horse, Staphylococcus aureus, Staphylococcus pseudintemedius, Staphylococcus epidermidis, Staphylococcus chromogens, Antimicrobial resistance, Multi-drug resistance, MDR, Gauteng, South Africa

\footnotetext{
* Correspondence: aodoi@utk.edu

'Department of Agriculture and Animal Health, College of Agriculture and

Environmental Sciences, University of South Africa, Florida Science Campus,

Johannesburg, South Africa

${ }^{3}$ Biomedical and Diagnostic Sciences, College of Veterinary Medicine,

University of Tennessee, Knoxville, USA

Full list of author information is available at the end of the article
} 


\section{Background}

Staphylococcus are Gram-positive cocci that comprise of over 50 species and subspecies, some of which are common commensals of various body sites of different animals [1, 2]. Although many Staphylococcus spp., are of no clinical significance, some are important opportunistic pathogens $[1,3,4]$. In equine medicine $S$. aureus, S. intermedius and S. hyicus, have been associated with clinical infections [2]. From a public health point of view, there are increasing reports of highly virulent staphylococcal infections that can be transmitted between horses and humans [5-7]. For example, a Canadian study reported that $96 \%$ of the Staphylococcus samples from horses and 93\% from humans displayed similar genetic profiles [8]. Another study done in the Netherlands reported that $S$. aureus isolated from a 16 year old girl was genetically similar to that isolated from a horse [5]. Busscher et al. [3] also reported identical Methicillin Resistant Staphylococcus aureus (MRSA) from horses and their caregivers.

Infections with antimicrobial drug resistant Staphylococcus spp. in both equine and human medicine has been associated with high morbidity, mortality and treatment costs. In animals, infections with antimicrobial drug resistant Staphylococcus spp. has also been associated with significant animal welfare implications due to animals staying sick for long periods in event of treatment failures [9-12]. Although coagulase-positive staphylococci (CoPS) are the most important groups associated with severe infections, coagulase-negative staphylococci (CoNS) have emerged as important pathogens as well. Moreover, all Staphylococcus spp., regardless of their coagulase activity, have potential to develop resistance to different classes of antimicrobials used for human and animal treatment [13]. Just like CoPS, resistance to antimicrobials such as gentamycin, macrolides, tetracycline, streptomycin, trimethoprim, sulfamethoxazole and fluoroquinolones is commonly observed among CoNS isolates from pets and horses $[13,14]$.

Excessive use of broad spectrum antimicrobials has been hypothesized as the main driver of antimicrobial drug resistance in Staphylococcus spp. [15]. For example, Bagcigil et al. [16] reported high levels of resistance to multiple antimicrobial agents including $\beta$-lactams in horses with previous history of treatment with $\beta$-lactams. Failure to complete the course of antimicrobial treatment has also been identified as a risk factor for development of resistance among staphylococcal isolates from horses [17]. Recent studies show that colonization with Staphylococcus spp. carrying antimicrobial resistance genes increases the risk of infection with resistant Staphylococcus [2, 17]. Transfer of resistance genes between pathogenic organisms and commensal flora has also been hypothesized as a risk factor for infection with resistant isolates [18]. Morton et al. [19] were able to demonstrate horizontal transfer of one conjugate mupirocin plasmid by finding the same plasmid in different staphylococcal isolates from patients in different areas of the hospital, which suggested that isolates had acquired new genes. Moreover, conjugative plasmids can move between CoPS and CoNS [19]. Furthermore, molecular epidemiological analyses by pulsedfield gel electrophoresis has shown that horizontal transfer of plasmid borne genes within and between different equine staphylococcal species is possible [20].

Despite the existence of evidence suggesting that horses play a significant role as sources of staphylococcal infections for humans $[5,6]$, it is surprising that studies of staphylococcal infections in Africa in general, and South Africa in particular, have mainly focussed on humans [12, 21, 22]. Available studies in human medicine suggest a high prevalence of resistant staphylococcal infections, especially MRSA, associated with both hospital and community acquired infections [9]. Therefore, studies of horses are critically needed to fill this knowledge gap. Given the paucity of data on both the burden of staphylococcal infections and the antibiotic resistance profiles among Staphylococcus isolates from horses in Africa, the present study is an exploratory descriptive investigation of the burden of staphylococcal infections and their antimicrobial drug resistance patterns among horses presented at the University of Pretoria veterinary teaching hospital from 2007 to 2012. This exploratory investigation is expected to provide useful information to guide future hypothesis driven studies in this region of the world.

\section{Methods}

\section{Study area}

This study was conducted using retrospective laboratory data collected from Gauteng province in South Africa. Gauteng province is approximately $18,178 \mathrm{~km}^{2}$ in size, and has an estimated population of 13.2 million people (24\% of the South African population). During the time period covered by this study, the province had a total of five metropolitan municipalities: Ekurhuleni, Sedibeng, Johannesburg, Tshwane and West Rand. The province has a subtropical climate, and is cooler in Johannesburg and slightly warmer in Pretoria. Gauteng is located in the Highveld region of South Africa, and has an annual summer rainfall of approximately $700 \mathrm{~mm}$. It has four seasons: summer (November-March), autumn (April-May), winter (June-August) and spring (September-October). Winter is the driest season, while December and January are the wettest months. The province experiences annual maximum temperatures of about $22{ }^{\circ} \mathrm{C}$ in the south and $25{ }^{\circ} \mathrm{C}$ in the north $[23,24]$. 


\section{Data source}

Laboratory records of all 1027 clinical samples from horses, from Gauteng Province, presented at the University of Pretoria bacteriology diagnostic laboratory for isolation and susceptibility testing of Staphylococcus spp. from January 2007 to December 2012 were included in the study. The data were received as paper records, reviewed and entered into an electronic database. The following fields were extracted for each record: horse breed, sex, age (in months), date sample was submitted as well as culture and antimicrobial susceptibility test results. The data were assessed for duplicate entries and if any horses had been sampled multiple times during the study period. No duplicates were identified. The dataset did not contain multiple tests from the same horse, neither were there mixed infections in the samples analysed. The breeds of horses were re-classified to identify the top 10 breeds. All other breeds that had small numbers were grouped into one category and called other breeds.

\section{Isolation of Staphylococcus spp. and testing for antimicrobial susceptibility}

The Bacteriology Laboratory at the University of Pretoria veterinary teaching hospital, from where the study data were obtained, follows standardized protocols for isolation of Staphylococcus spp. based on the methods described in Quinn et al. [25]. Susceptibility testing of samples was conducted using the Kirby Bauer disc diffusion technique following the guidelines described in the Clinical and Laboratory Standards Institute (CLSI) document [26]. The isolates were subjected to antimicrobial susceptibility testing against a panel of 9 drugs using the disc diffusion method. Antimicrobial resistance (AMR) was defined as resistance to at least one antimicrobial while multidrug resistance (MDR) was defined as resistance to 3 or more classes of antimicrobials [27]. Thus, some of the isolates classified as AMR were also included in the MDR group if they exhibited resistance to 3 or more classes of antimicrobials.

\section{Statistical analysis}

Shapiro-Wilks test [28] was used to test normality of continuous variables. Non-normally distributed continuous variables were summarized using medians and interquartile ranges. Age was categorized into four categories: foals ( $<1$ year old), yearlings (1-2 years old), fillies and colts (2-4 years old) and adults ( $>4$ years old). Crude and factor-specific percentages of Staphylococcus positive samples, AMR and MDR isolates as well as their 95\% confidence intervals were computed. The factors (categorical variables) considered were: species of Staphylococcus, geographic location, season, year, breed, age group and sex. Associations between these categorical variables and percentages of Staphylococcus positive samples, AMR and
MDR isolates were assessed using Chi-square and, in cases of small expected cell sizes, Fishers Exact test. Significance was assessed at $p \leq 0.05$. Due to the small sample sizes involved in this exploratory descriptive investigation, adjusted associations using multiple regression models could not be assessed. All statistical analyses were performed in STATA [29].

\section{Results}

A total of 1027 clinical samples from horses in Gauteng Province were submitted to the University of Pretoria bacteriology diagnostic laboratory from 2007 to 2012 and were included in this study. Of the 1027 processed samples, $12.0 \%(123 / 1027)$ were positive for staphylococci, the majority of which were $S$. aureus (41.5\%) followed by $S$. pseudintermedius (14.6\%), S. epidermidis (4.9\%), S. equinus (0.8\%), and S. chromogens (0.8\%). The remaining $37.4 \%$ of the samples did not have species information. Of the Staphylococcus positive samples, a total of $52.0 \%(64 / 123)$ had isolates that exhibited antimicrobial resistance to at least one antimicrobial (AMR), while $28.5 \%(35 / 123)$ had isolates that exhibited multidrug resistance (MDR). Significantly $(p=0.002)$ more samples had isolates that exhibited AMR (52.0\%; 95\% CI: 42.8-61.1) than those that had MDR (28.5\%; 95\% CI: 20.7-37.3\%).

\section{Distribution by animal characteristics}

The samples that were submitted and processed, came from 29 different breeds of horses. The five most common breeds contributing samples were: Thoroughbreds (26.8\%), European warm blood (12.6\%), Arab (11.6\%), South African warm blood (6.4\%) and Friesian (6.0\%) (Table 1). A significant $(p=0.001)$ association was observed between breed and the percentage of Staphylococcus positive samples, with the highest percentage of Staphylococcus positive samples observed among crossbreeds (20.5\%), followed by Boerperds (18.9\%) and European breeds (17.8\%). There was also a significant ( $p=0.015)$ association between breed of the horse and the percentage of samples carrying resistant isolates, with the highest percentage of samples with resistant isolates being observed among the Boerperds (85.7\%) followed closely by the American saddler (75\%) and the European warm blood (73.9\%). Similarly, there was a significant $(p=0.006)$ association between the percentage of samples carrying MDR isolates and horse breed, with the American Saddler having the highest percentage (75\%) of MDR followed by European warm blood (56.5\%), Thoroughbreds (33.3\%) and the Boerperds (28.6\%).

The median age of the horses was 4.3 years, but ranged from 0 to 25.7 years (interquartile range: $0.7-$ 9 years). The majority of the samples were from adults (50.6\%), followed by foals $(27.1 \%)$. Yearlings contributed 
Table 1 Host factor distribution of equine samples from Gauteng Province (South Africa) tested for Staphylococcus, 2007-2012

\begin{tabular}{|c|c|c|c|c|c|c|}
\hline \multirow[b]{2}{*}{ Breed } & \multicolumn{3}{|c|}{$\begin{array}{l}\text { All Samples Processed } \\
(n=1027)\end{array}$} & \multicolumn{3}{|c|}{$\begin{array}{l}\text { Staphylococcus Positive Samples } \\
(n=123)\end{array}$} \\
\hline & $\mathrm{n}^{\mathrm{a}}$ & $\%^{\mathrm{b}}$ & $95 \% \mathrm{Cl}^{\mathrm{C}}$ & $\mathrm{n}^{\mathrm{a}}$ & $\%^{\mathrm{b}}$ & $95 \% \mathrm{Cl}^{\mathrm{C}}$ \\
\hline American Saddler & 39 & 3.8 & $2.8,5.2$ & 4 & 10.3 & $3.8,24.6$ \\
\hline Arab & 119 & 11.6 & $9.8,13.7$ & 8 & 6.7 & $3.4,12.9$ \\
\hline Boerperd & 37 & 3.6 & $2.6,4.9$ & 7 & 18.9 & $9.2,35.0$ \\
\hline Crossbreed & 39 & 3.8 & $2.8,5.2$ & 8 & 20.5 & $10.5,36.2$ \\
\hline European warm blood & 129 & 12.6 & $10.7,14.7$ & 23 & 17.8 & $12.1,25.4$ \\
\hline Friesian & 62 & 6.0 & $4.7,7.7$ & 2 & 3.2 & $0.8,12.1$ \\
\hline Nooitgedachtpony & 43 & 4.2 & $3.1,5.6$ & 5 & 11.6 & $4.9,25.3$ \\
\hline South African warm blood & 66 & 6.4 & $5.1,8.1$ & 1 & 1.5 & $0.2,10.1$ \\
\hline Thoroughbred & 275 & 26.8 & $24.2,29.6$ & 21 & 7.6 & $5.0,11.4$ \\
\hline Welshpony & 25 & 2.4 & $1.6,3.6$ & 3 & 12.0 & $3.8,31.9$ \\
\hline Percheron & 13 & 1.3 & $0.7,2.2$ & 0 & 0 & - \\
\hline All other breeds & 73 & 7.1 & $5.6,8.9$ & 6 & 8.2 & $3.1,17.1$ \\
\hline Unspecified or missing & 107 & 10.4 & $8.7,12.4$ & 35 & 32.7 & $24.0,42.5$ \\
\hline \multicolumn{7}{|l|}{ Age Group } \\
\hline Foal $(<1$ year $)$ & 278 & 27.1 & $24.4,29.9$ & 58 & 20.9 & $16.2,26.1$ \\
\hline Yearling (1-2 years) & 83 & 8.1 & $6.5,9.9$ & 9 & 10.8 & $5.1,19.6$ \\
\hline Filly or Colt ( $2-4$ years) & 146 & 14.2 & $12.1,16.5$ & 13 & 8.9 & $4.8,14.7$ \\
\hline Adult ( $>4$ years) & 520 & 50.6 & $47.5,53.7$ & 43 & 8.3 & $6.0,11.0$ \\
\hline \multicolumn{7}{|l|}{ Sex } \\
\hline Males & 499 & 48.6 & $45.5,51.7$ & 48 & 9.6 & $7.2,12.6$ \\
\hline Females & 434 & 42.2 & $39.2,45.3$ & 44 & 10.1 & $7.6,13.4$ \\
\hline Unspecified or missing & 94 & 9.2 & $7.5,11.1$ & 31 & 33.0 & $23.6,43.4$ \\
\hline
\end{tabular}

${ }^{a}$ number of samples

bercentage of samples

c95\% confidence interval

the lowest percentage of samples (8.1\%) (Table 1). A significant $(p=0.001)$ association was observed between age group and percentage of Staphylococcus positive samples. Foals had the highest percentage (20.9\%; 95\% CI: 16.2, 26.1\%) of Staphylococcus positive samples followed by yearlings $(10.8 \%$; $95 \%$ CI: $5.1,19.6)$, fillies/ colts $(8.9 \%$; 95\% CI: 4.8, 14.7\%) and adults (8.3\%; 95\% CI: 6.0, 11.0\%). However, there was no significant difference $(p=0.360)$ in the levels of antimicrobial resistance across age groups. On the contrary, a significant $(p=0.012)$ association in the levels of samples that had MDR isolates was observed across age groups, with fillies/colts having the highest levels (46.2\%; 95\% CI: 19.2, $74.9 \%$ ) followed by adults (42.0\%; 95\% CI: 27.0, 57.9\%), foals $(17.2 \%$; $95 \%$ CI: $8.6,29.4 \%)$ and yearlings had the lowest (11.1\%; 95\% CI: 0.2, 48.2\%).

Significantly $(p=0.001)$ more samples from males (48.6\%) than from females (42.2\%) were processed. However, there were no significant $(p=0.438)$ differences in the percentages of Staphylococcus positive samples obtained from males (9.6\%) and females $(10.1 \%)$ (Table 1$)$. Similarly, no significant $(p=0.231)$ differences in the percentage of samples with resistant isolates were observed between males (64.6\%); 95\% CI: 49.5, 77.8\%) and females (52.3\%; 95\% CI: 36.7, $67.5 \%)$. Interestingly, there was a significantly $(p=0.024)$ higher percentage of MDR isolates among samples from male $(41.7 \%$; $95 \%$ CI: 27.6, 56.8) than female horses (20.5\%; 95\% CI: 9.8, 35.3\%).

\section{Temporal patterns}

The largest percentage of samples were submitted during summer $(40.8 \%)$ while the lowest $(15.6 \%)$ were submitted during the spring (Table 2). Although spring (14.4\%) and autumn (14.1\%) tended to have higher percentages of Staphylococcus positive samples than the other two seasons, there was no significant $(p=0.357)$ association between season and percentage of Staphylococcus positive samples (Table 2). However, a significant $(p=0.002)$ association between 
Table 2 Temporal and Geographic distribution of equine samples from Gauteng Province assessed for antimicrobial susceptibility, 2007-2012

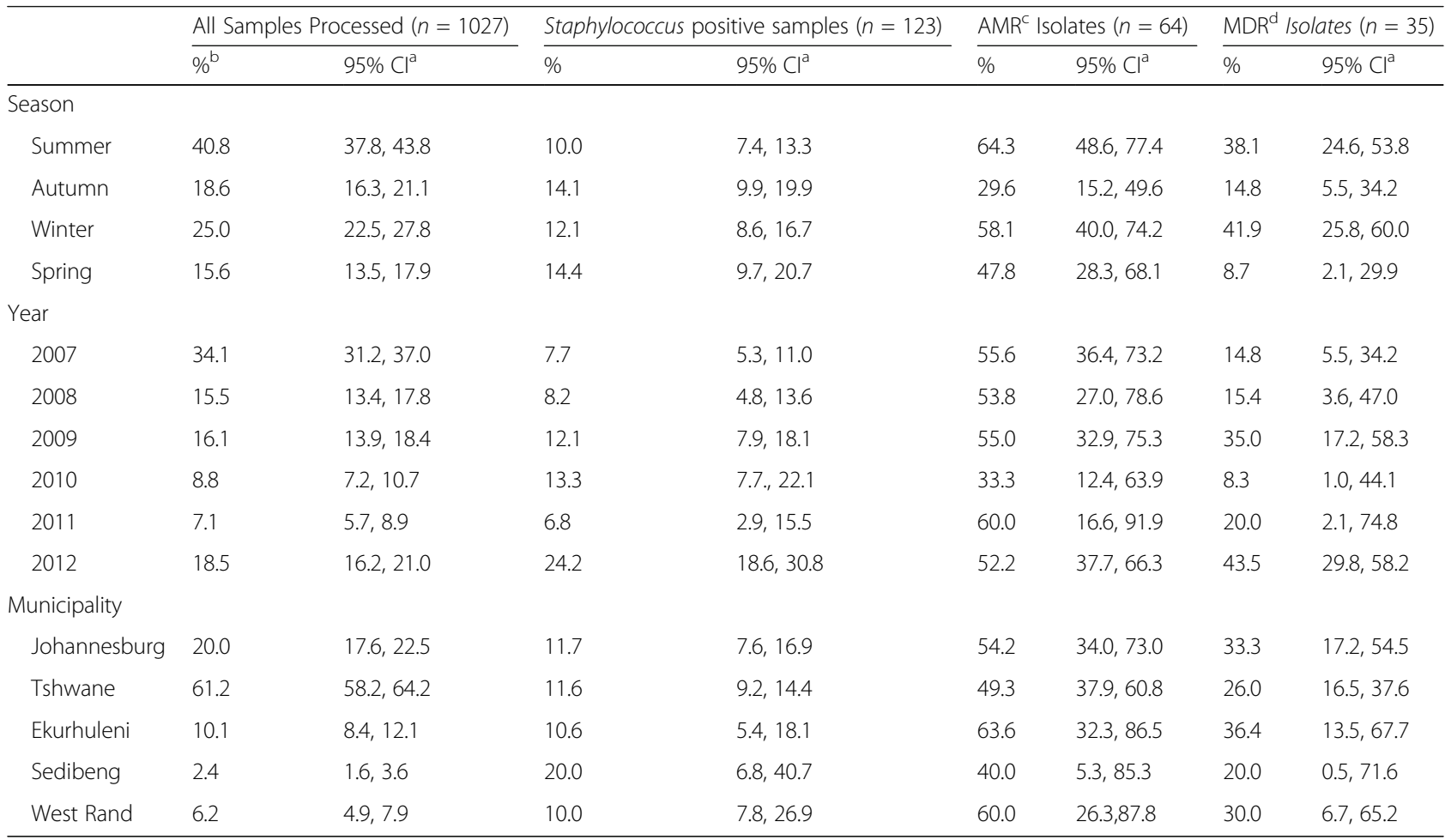

${ }^{\mathrm{a}} 95 \%$ confidence interval

bercent

CAMR: Antimicrobial resistant (defined as resistance to at least one antimicrobial)

${ }^{d}$ MDR: Multidrug resistant (defined as resistance to at least three classes of antimicrobials; includes a subset of AMR isolates)

season and percentage of isolates resistant to at least one antimicrobial was observed. Summer had the highest $(64.3 \%$; 95\% CI: 48.6, 77.4\%) and autumn the lowest $(29.6 \%$; $95 \%$ CI: $15.2,49.6 \%)$ percentage of antimicrobial resistant isolates (Table 2). On the contrary, there was no significant $(p=0.137)$ association between the percentage of MDR isolates and season.

A comparison of the percentage of samples submitted and processed each year revealed that significantly more samples were processed in 2007; and the lowest percentage of samples were received and processed in 2011. Moreover, there was a significant $(p<0.001)$ association between year and the percentage of Staphylococcus positive samples, with the largest percentage of positive samples occurring in 2012 (24.2\%) (Table 2). Thus, except for the anomaly observed in 2011, there was an increasing trend in the percentage of Staphylococcus positive samples during the study period (Table 2). By contrast, there were no significant $(p=0.707)$ differences in the percentage of AMR isolates across the years, neither were there significant differences $(p=0.304)$ in the percentage of MDR isolates across the years.

\section{Geographic patterns}

The geographic distribution of sample submissions was a reflection of the distribution of horse populations across the different municipalities with Tshwane submitting most (61.2\%) of the samples followed by Johannesburg (20\%) (Table 2). Although the percentage of Staphylococcus positive samples varied from $10 \%$ in Ekurhuleni municipality to $20 \%$ in Sedibeng municipality (Table 2), these differences were not statistically significant $(p=0.563)$. The percentage of isolates that were resistant to at least one antimicrobial varied from $40 \%$ in Sedibeng to $63.6 \%$ in Ekurhuleni (Table 2). Again, there were no significant $(p=0.857)$ differences in the percentage of isolates that were resistant to at least one antimicrobial. Similarly, the percentage of MDR isolates varied from 20\% in Sedibeng to $36.4 \%$ in Ekurhuleni municipality (Table 2). As was the case with AMR isolates, there were no significant $(p=0.891)$ differences in the percentage of MDR isolates across the municipalities.

\section{Antimicrobial susceptibility profiles}

There was a significant $(p<0.001)$ difference in the percentages of samples with resistant isolates across the 
Staphylococcus spp., with S. aureus (72.5\%) showing much higher levels of resistance than $S$. pseudintermedius (38.9\%) (Table 3). Similarly, there was a significant $(p=0.0001)$ difference in the percentages of samples with MDR isolates across species, and again $S$. aureus (52.9\%) exhibited higher levels of MDR compared to $S$. pseudintermedius (16.7\%) (Table 3).

Significant $(p<0.05)$ differences in the levels of resistance to each antimicrobial was also observed, with resistance to $\beta$-lactams being the highest (ampicillin: 84.5\%; penicillin: $74.1 \%$ ) followed by Trimethoprim/sulfamethoxazole (folate pathway inhibitors) $(60.9 \%)$, while the lowest level of resistance was towards amikacin (14.1\%) (Table 3). Most MDR isolates tended to exhibit resistance to trimethoprim/sulfamethoxazole (53.1\%), followed by gentamicin (48.9\%) (Table 3). Only $9.1 \%$ of these isolates

Table 3 Distribution of antimicrobial resistance by species of Staphylococcus and antimicrobial agent

\begin{tabular}{|c|c|c|c|c|}
\hline & \multicolumn{2}{|c|}{ Resistant Isolates } & \multicolumn{2}{|c|}{ MDR Isolates } \\
\hline & $\%$ & $95 \% \mathrm{Cl}$ & $\%$ & $95 \% \mathrm{Cl}$ \\
\hline Species & & $n=123$ & & $n=35$ \\
\hline S. aureus & 72.5 & $58.3,83.3^{\mathrm{a}}$ & 52.9 & $38.5,67.1^{\mathrm{a}}$ \\
\hline S. pseudintermedius & 38.9 & $19.2,63.0^{b}$ & 16.7 & $3.6,41.4^{b}$ \\
\hline S. $s p p^{\prime}$ & 30.4 & $18.7,45.4^{\mathrm{b}}$ & 2.2 & $0.1,11.5^{c}$ \\
\hline All other species ${ }^{2}$ & 75.0 & $34.9,96.8^{\mathrm{a}}$ & 50.0 & $15.7,84.3^{\mathrm{a}}$ \\
\hline Antimicrobial \& Antimicrobial Class & & $n=64$ & & $n=35$ \\
\hline \multicolumn{5}{|l|}{ Aminoglycosides $^{3}$} \\
\hline Gentamycin & 54.6 & $42.1,66.7^{\mathrm{a}}$ & 48.9 & $38.1,59.8$ \\
\hline Amikacin & 14.1 & $7.3,25.2^{\mathrm{e}}$ & 9.1 & $4.0,17.1$ \\
\hline \multicolumn{5}{|l|}{ B-lactams ${ }^{4}$} \\
\hline Ampicillin & 84.5 & $72.3,91.9^{b}$ & 37.5 & $27.4,48.5$ \\
\hline Penicillin & 74.1 & $61.0,84.0^{\mathrm{b}}$ & 37.5 & $27.4,48.5$ \\
\hline Ceftriaxone & 44.8 & $32.3,58.1^{c}$ & 30.0 & $20.3,41.3$ \\
\hline \multicolumn{5}{|l|}{ Fluoroquinolones ${ }^{5}$} \\
\hline Enrofloxacin & 23.4 & $14.5,35.7^{c}$ & 18.8 & $10.1,30.5^{b}$ \\
\hline \multicolumn{5}{|l|}{ Folate Pathway Inhibitors ${ }^{6}$} \\
\hline Trimotherim-sulfamethoxazole & 60.9 & $48.2,72.3^{\mathrm{a}}$ & 53.1 & $40.2,65.7^{\mathrm{a}}$ \\
\hline \multicolumn{5}{|l|}{ Tetracyclines $^{7}$} \\
\hline Doxycyline & 46.9 & $34.8,59.4^{\mathrm{a}, \mathrm{d}}$ & 35.9 & $24.3,48.9^{c}$ \\
\hline \multicolumn{5}{|l|}{ Phenicols } \\
\hline Chloramphenicol & 44.1 & $31.7,57.2^{d}$ & 31.3 & $20.2,44.1^{c}$ \\
\hline
\end{tabular}

${ }^{1}$ Samples that were not identified to species level and were thus reported as Staphylococcus spp.

${ }^{2}$ Other species included S. equinus, S. chromogens and S. epidermidis

${ }^{3}$ Gentamycin and amikacin

${ }^{4}$ Penicillin, ampicillin, ceftriaxone

${ }^{5}$ Enrofloxacin

${ }^{6}$ Sulphamethoxazole

${ }^{7}$ Chloramphenicol

${ }^{a-d}$ Estimates with different superscripts are significantly different at $5 \%$

significance level exhibited resistance towards amikacin and only $18.8 \%$ displayed resistance towards enrofloxacin. The distribution of the number of classes of antimicrobials to which isolates were resistant is shown in Table 4.

The majority of MDR combinations observed involved S. aureus (Table 5). Multidrug resistance involving combinations of four antimicrobials tended to occur more frequently in young foals as compared to combinations that involved more antimicrobials. Most samples with MDR isolates came from Tshwane municipality (Table 5) and involved mainly European warm blood (31.1\%) and Thoroughbreds (20.0\%). It is interesting to note that a high percentage (35.6\%) of MDR isolates were from horses less than 1 month old (Table 5).

\section{Discussion}

The current study is an exploratory descriptive analysis of antimicrobial resistance patterns of Staphylococcus spp. isolated from horses presented at a veterinary teaching hospital in South Africa. Since very little is known regarding the epidemiology of antimicrobial resistance in horses in Africa and many other developing economies, this study is intended to provide preliminary information to guide future more detailed hypothesis driven epidemiological studies of antimicrobial drug resistance among Staphylococcus spp. not only in horses, but other domestic species as well.

Contrary to findings by Leekha et al. [30] the present study did not find significant seasonal differences in the percentage of Staphylococcus positive samples. This could be due to aggregation of cases by pre-defined seasons, as was done in the present study. Other authors have argued that this aggregation leads to loss of information if infection occurrences are not seasonal but follow other cyclical patterns such as biannual patterns [30]. Some authors have suggested use of time-series analytical approaches in such situations [31]. Unfortunately, due to the exploratory nature and the small samples sizes of the current study, time-series analysis could not be performed. The significant association observed between season and percentage of antimicrobial resistance could be attributed to weather

Table 4 Distribution of number of antimicrobial classes to which equine samples exhibited resistance $(n=64)$

\begin{tabular}{llll}
\hline $\begin{array}{l}\text { Number of antimicrobial classes } \\
\text { to which isolates exhibited resistance }\end{array}$ & $\begin{array}{l}\text { Number } \\
\text { of isolates }\end{array}$ & Percent & $95 \% \mathrm{Cl}^{\mathrm{a}}$ \\
\hline 1 & 17 & 26.6 & $16.3,39.1$ \\
2 & 12 & 18.8 & $10.1,30.4$ \\
3 & 7 & 10.9 & $4.5,21.2$ \\
4 & 12 & 18.8 & $10.1,30.4$ \\
5 & 8 & 12.5 & $5.6,23.2$ \\
6 & 8 & 12.5 & $5.6,23.2$ \\
\hline${ }^{a} 95 \%$ confidence interval & & &
\end{tabular}


Table 5 Antimicrobial resistance patterns of staphylococcal isolates from equine samples from Gauteng Province (South Africa), 2007-2012

\begin{tabular}{|c|c|c|c|c|c|}
\hline $\begin{array}{l}\text { Horse Age } \\
\text { (months) }\end{array}$ & Breed of Horse & Municipality & Year & Staphylococcus Species & Antimicrobial Resistance Patterns ${ }^{\text {a }}$ \\
\hline 0 & Thoroughbred & Tshwane & 2008 & S. aureus & AMP-DOX-PEN \\
\hline 0 & Europeanwarmblood & Mogale City & 2011 & Staph. spp. & AMP-PEN-SUL \\
\hline 132 & Crossbreed & Tshwane & 2007 & S. aureus & AMI-AMP-PEN \\
\hline 0 & Boerperd & Tshwane & 2007 & S.pseudintermedius & AMP-DOX-PEN \\
\hline 0 & Boerperd & Tshwane & 2007 & S.aureus & AMP-DOX-PEN \\
\hline 0 & Unpecified or Missing & Tshwane & 2007 & Staph. spp. & AMP-CEF-PEN \\
\hline 131 & Europeanwarmblood & Johannesburg & 2008 & S. aureus & AMP-PEN-SUL \\
\hline 48 & Europeanwarmblood & Tshwane & 2012 & S.aureus & AMP-GEN-PEN-SUL \\
\hline 182 & Boerperd & Tshwane & 2012 & S.aureus & AMP-GEN-PEN-SUL \\
\hline 1 & Arab & Tshwane & 2007 & S. equinus & AMP-CEF-PEN-SUL \\
\hline 96 & All other breeds & Tshwane & 2012 & S. epidermidis & DOX-ENR-GEN-SUL \\
\hline 40 & All other breeds & Tshwane & 2007 & S. aureus & AMP-CEF-GEN-PEN-SUL \\
\hline 96 & Europeanwarmblood & Tshwane & 2012 & S. epidermidis & DOX-ENR-GEN-PEN-SUL \\
\hline 0 & Unpecified or Missing & Tshwane & 2012 & S. aureus & AMP-CEF-CHL-PEN-SUL \\
\hline 83 & Europeanwarmblood & Johannesburg & 2012 & S. aureus & AMP-CHL-GEN-PEN-SUL \\
\hline 48 & Europeanwarmblood & Tshwane & 2012 & S. aureus & AMP-DOX-GEN-PEN-SUL \\
\hline 84 & Thoroughbred & Tshwane & 2012 & S. aureus & AMP-CEF-GEN-PEN-SUL \\
\hline 0 & Europeanwarmblood & Johannesburg & 2008 & S. aureus & AMP-CEF-GEN-PEN-SUL \\
\hline 0 & Unpecified or Missing & Tshwane & 2012 & S. aureus & AMP-CEF-CHL-GEN-PEN-SUL \\
\hline 0 & Unpecified or Missing & Emfuleni & 2012 & S. aureus & AMP-CEF-CHL-GEN-PEN-SUL \\
\hline 0 & Unpecified or Missing & Tshwane & 2012 & S. aureus & AMP-CEF-DOX-GEN-PEN-SUL \\
\hline 84 & Thoroughbred & Ekurhuleni & 2007 & S. aureus & AMP-CEF-DOX-GEN-PEN-SUL \\
\hline 168 & Europeanwarmblood & Ekurhuleni & 2012 & S. aureus & AMP-CEF-CHL-GEN-PEN-SUL \\
\hline 0 & American Saddler & Ekurhuleni & 2007 & S. aureus & AMP-CEF-DOX-GEN-PEN-SUL \\
\hline 81 & Thoroughbred & Tshwane & 2009 & S. aureus & AMP-CEF-DOX-GEN-PEN-SUL \\
\hline 0 & American Saddler & Ekurhuleni & 2007 & Staph. spp. & AMP-CEF-DOX-GEN-PEN-SUL \\
\hline 48 & Europeanwarmblood & Westonaria & 2012 & S. aureus & AMP-CHL-DOX-GEN-PEN-SUL \\
\hline 19 & Europeanwarmblood & Westonaria & 2012 & S. aureus & AMP-CHL-DOX-ENR-GEN-PEN-SUL \\
\hline 108 & Thoroughbred & Tshwane & 2009 & S. aureus & AMP-CEF-CHL-DOX-GEN-PEN-SUL \\
\hline 0 & Arab & Johannesburg & 2012 & S. aureus & AMI-AMP-CHL-DOX-GEN-PEN-SUL \\
\hline 168 & Europeanwarmblood & Johannesburg & 2012 & S. aureus & AMP-CEF-CHL-DOX-GEN-PEN-SUL \\
\hline 36 & Europeanwarmblood & Westonaria & 2010 & S.pseudintermedius & AMP-CEF-CHL-DOX-ENR-GEN-SUL \\
\hline 111 & All other breeds & Tshwane & 2009 & S.pseudintermedius & AMP-CEF-CHL-DOX-GEN-PEN-SUL \\
\hline 96 & American Saddler & Tshwane & 2012 & S.aureus & AMI-AMP-CEF-CHL-GEN-PEN-SUL \\
\hline 0 & Nooitgedachtpony & Tshwane & 2009 & S. aureus & AMI-AMP-CEF-CHL-DOX-ENR-GEN-PEN \\
\hline 120 & Europeanwarmblood & Johannesburg & 2009 & S.epidermidis & AMI-AMP-CEF-DOX-ENR-GEN-PEN-SUL \\
\hline 182 & Boerperd & Tshwane & 2012 & S.aureus & AMP-CEF-CHL-DOX-ENR-GEN-PEN-SUL \\
\hline 48 & Europeanwarmblood & Tshwane & 2012 & S.epidermidis & AMP-CEF-CHL-DOX-ENR-GEN-PEN-SUL \\
\hline 155 & Thoroughbred & Johannesburg & 2012 & S.aureus & AMI-AMP-CHL-DOX-ENR-GEN-PEN-SUL \\
\hline 83 & Thoroughbred & Johannesburg & 2011 & S.pseudintermedius & AMI-AMP-CEF-CHL-DOX-ENR-GEN-PEN-SUL \\
\hline 0 & Unpecified or Missing & Tshwane & 2009 & S.aureus & AMI-AMP-CEF-CHL-DOX-ENR-GEN-PEN-SUL \\
\hline 72 & Thoroughbred & Tshwane & 2009 & S.aureus & AMI-AMP-CEF-CHL-DOX-ENR-GEN-PEN-SUL \\
\hline
\end{tabular}

${ }^{\mathrm{a}} \mathrm{AMI}=$ Amikacin; $\mathrm{AMP}=$ Ampicillin; $\mathrm{CEF}=$ Ceftriaxone; $\mathrm{CHL}=$ Chloramphenicol; DOX = Doxycycline; ENR = Enrofloxacin; GEN = Gentamicin; PEN = Penicillin; SUL = Sulphametho 
conditions such as temperature and humidity. However, it may also be due to seasonal differences in staffing levels (i.e., fewer regular staff during vacation months) and seasonal differences in antimicrobial drug use due to differences in infection rates from other bacteria resulting in differences in selection pressure [30].

Although the percentages of Staphylococcus positive samples increased over time, there were no similar increases in the number of samples submitted for processing. Therefore, the observed increases in percentage of Staphylococcus positive samples may be a reflection of a true increase in the number of staphylococcal infections over the years. However, more detailed primary base studies will need to be performed to further investigate this trend. The higher levels of Staphylococcal infections in some breeds of horses seem to suggest potential breed predisposition. However, the reason for this is unclear and no previous studies have reported this. Therefore, this warrants further investigations to establish the potential existence of breed predisposition. Although sex dimorphism of staphylococcal infections, especially methicillin resistant infections, have been observed in humans [32], no similar associations were observed in this study and to our knowledge, no studies have reported differences in staphylococcal infections in horses based on sex.

Coagulase positive Staphylococcus spp., especially $S$. aureus are responsible for a significantly large percentage of infections in horses as opposed to coagulase negative strains [1]. Therefore, the higher levels of resistance observed among $S$. aureus in this study is of concern, from a therapeutic point of view, due to the resulting potential treatment failures associated with the observed high levels of resistance. The relatively high levels of AMR and MDR observed in this study may suggest high selection pressure among Staphylococcus spp. isolates from horses treated at the veterinary teaching hospital. This may be due to the fact that most of the cases seen in the teaching hospital tend to be referral cases that may not have responded well to initial antimicrobial treatments by the primary care veterinarians. Thus, it is possible that most of these horses would have been on antibiotic treatment for prolonged periods before being transferred to the referral veterinary teaching hospital. Another plausible explanation for the high levels of antimicrobial resistance observed in the study could be the result of failure of horse owners to ensure that the animals complete the full course of prescribed antimicrobial therapy. This issue has been highlighted by some authors who reported that caretakers have a tendency to stop drug administration as soon as disease symptoms abate, a practice that has been incriminated in the development of antimicrobial drug resistance [17]. Transportation stress has also been incriminated in the high carriage of resistant Staphylococcus in equine patients admitted to clinics as a result of direct contact with referring veterinarians who might be carrying resistant Staphylococcus [4].

The high levels of antimicrobial resistance observed in this study is consistent with the observations in humans in South Africa by Essa et al. [12], who observed that up to $95.1 \%$ of the samples were MDR, and only $3.7 \%$ of the samples were susceptible to all antibiotics tested in the study. The very high levels of resistance to $\beta$-lactams observed in the current study is consistent with reports by Weese [1] who reported high levels of resistance to $\beta$-lactams among staphylococci and especially S. aureus. Some studies have reported that routine use of $\beta$-lactam antibiotics in prevention of surgical infections, predisposes horses to acquisition of methicillin resistant $S$. aureus [33, 34]. Therefore, the high levels of resistance to $\beta$-lactam antibiotics in the present study could signal the existence of methicillin resistance in staphylococcal infections in horses presented at the hospital under study. Unfortunately, methicillin was not routinely included in the susceptibility test panels used by the veterinary teaching hospital that supplied the data for the current study, and hence the levels of resistance to methicillin could not be assessed in the current study. However, it is encouraging to note that resistance to fluoroquinolones was relatively low implying that these antimicrobials are still relatively effective and therefore their use could more likely be associated with successful treatment outcomes compared to $\beta$-lactams. It is worth noting that in South Africa, some of the older antibiotics are readily available to farmers over the counter while the newer ones require prescription [35]. This may have impacted selection pressure for antimicrobial resistance especially influencing antimicrobials readily available over the counter.

The observed low levels of resistance towards aminoglycosides was not unusual. For instance, Abrahamsen [17] in a study conducted in Maine (USA), also observed that Staphylococcus spp. tended to be susceptible to both gentamicin and amikacin, both of which are aminoglycosides. Furthermore, a study by Schnellmann et al. [33], investigating emergence of resistance after hospitalization in Switzerland, found much lower levels of resistance to amikacin compared to other antimicrobials. However, it is worth noting that the level of resistance observed here may not be a true reflection of the level of resistance in the larger horse population. For example, Van den Eede et al. [4] observed a much lower level of resistance among horses on farms that were situated in a region surrounding an equine clinic in Belgium whereas much higher carriage rates of resistant Staphylococcus had been detected in horses presented at the clinic [4]. 
The significant differences in the percentage of MDR isolates across species, with $S$. aureus exhibiting higher levels of MDR compared to other species, was anticipated given that it is the most common species in horses and therefore more likely to be exposed to higher selection pressure during antibiotic treatments compared to the other less common species [30]. Worth noting was that MDR was frequently observed among isolates from horses less than 1 month old. This could be due to the fact that foals frequently require antimicrobial treatment to combat a variety of conditions. Moreover, the basic principle of antimicrobial treatment in neonatal foals is the use of broad-spectrum antimicrobials [36]. This practice potentially increases the selection pressure for antimicrobial resistance among foals. However, it is interesting to note that MDR isolates resistant to six or more drugs tended to occur in older horses, which suggests that the overall selection pressure for antimicrobial drug resistance could be higher in older horses. Some authors have indicated that after 3 days of hospitalization and treatment with penicillin, the percentage of Staphylococcus isolates showing antibiotic resistance dramatically increases in horses [33].

Although some authors have reported geographic differences in levels of AMR staphylococcal isolates [37], the current study did not identify significant geographic differences. This could most likely be due to the small samples sizes involved in some of the municipalities included in the study. Therefore, more detailed and larger studies need to be performed to more fully investigate geographic and other determinants of variations in AMR and MDR staphylococcal infections. Thus, our next set of studies will focus on these issues as well as investigating practices in the use of antimicrobials by both veterinary practitioners and animal owners.

A limitation of this retrospective laboratory-based study is that the source of samples (e.g. nasal, skin, etc) could not be assessed due to quality of these data. A positive outcome of this finding is that we will work with the laboratory to improve laboratory data capture. This will have a positive impact of improved data quality for similar studies in the future. Additionally, information on past antimicrobial use was not available and therefore we could not assess its associations with levels of AMR or MDR. Moreover, detailed analysis of the $\beta$-lactam resistant isolates were not performed and hence we could not assess their susceptibility to methicillin. Finally, it is possible that some of the isolates reported as Staphylococcus spp. may have belonged to $S$. aureus, S. pseudintermedius, S. epidermidis or S. equinus. Unfortunately, information was not available to elucidate this.

\section{Conclusions}

The above limitations notwithstanding, the study has shown evidence of seasonal patterns of staphylococcal infections and occurrence of AMR and MDR in horses. There is also evidence of differences in the occurrence of AMR and MDR by species of Staphylococcus and breed of horse. Resistance among Staphylococcus isolates was highest towards $\beta$-lactam antibiotics, but lowest towards amikacin. It should be pointed out that this was an exploratory descriptive study that did not lend itself to more detailed analyses such as use of multivariable models due to the small sample sizes involved. However, this being the first study of its kind in Africa, it provides useful descriptive information to guide future more detailed studies intended to address the problems of equine staphylococcal infections and antimicrobial resistance. Routine surveillance, prudent antimicrobial use and efficient infection control should be advocated as strategies to contain development of antimicrobial resistance in horse infections. Future studies will need to be primary-base involving large sample sizes to help understand specific local factors contributing to development of antimicrobial resistance so as to better guide treatment and control efforts.

\section{Abbreviations}

95\% Cl: 95\% Confidence Interval; AMR: Antimicrobial Resistant; CoNS: coagulase-negative staphylococci; CoPS: coagulase-positive staphylococci; MDR: Multidrug Resistant; MRSA: Methicillin Resistant Staphylococcus aureus; p: $p$-value; S. aureus: Staphylococcus aureus; S. epidermidis: Staphylococcus epidermidis; S. equinus: Staphylococcus equinus; $S$. hyicus: Staphylococcus hyicus; S. intermedius: Staphylococcus intermedius; S. pseudintermedius: Staphylococcus pseudintermedius; spp.: Species; USA: United States of America; $\beta$-lactam: Beta-lactam

\section{Acknowledgments}

Carnegie African Diaspora Program and the University of South African Visiting Research Program are gratefully acknowledged for making it possible for Dr. Odoi to travel to South Africa to conduct the study.

\section{Funding}

This project was made possible by funding from the University of Tennessee Centers of Excellence, Carnegie African Diaspora Fellowship Program and the University of South Africa visiting research program that funded the research collaboration between Drs Odoi and Oguttu. The funders had no role in study design, data collection and analysis, decision to publish, or preparation of the manuscript.

\section{Availability of data and materials}

The data that support the findings of this study are available from the bacteriology laboratory of the University of Pretoria that has legal ownership of the data. The data are not publicly available and can be requested for and obtained from the above listed legal owner.

\section{Authors' contributions}

JWO was involved in study design, results interpretation and editing of the manuscript. DNQ was involved in study design, data entry and manuscript writing. AO conceived the study idea, was involved in study design, analysed the data, interpreted the results and was involved in extensive editing of the manuscript. All authors read and approved the final manuscript

Ethics approval and consent to participate

This study was approved by the Animal Ethics Committees of both the University of Pretoria and the University of South Africa (reference number 
V051-14 and Ref. NR.: 2014/CAES/077 respectively). Consent for animal samples to be used in research and for the findings to be published was obtained from animal owners at the time they consented for their animals to receive care at the veterinary teaching hospital.

\section{Consent for publication}

Not applicable

\section{Competing interests}

The authors declare that they have no competing interests.

\section{Publisher's Note}

Springer Nature remains neutral with regard to jurisdictional claims in published maps and institutional affiliations.

\section{Author details}

${ }^{1}$ Department of Agriculture and Animal Health, College of Agriculture and Environmental Sciences, University of South Africa, Florida Science Campus, Johannesburg, South Africa. ${ }^{2}$ Section Veterinary Public Health, Department of Paraclinical Sciences, Faculty of Veterinary Sciences, University of Pretoria, Pretoria, South Africa. ${ }^{3}$ Biomedical and Diagnostic Sciences, College of Veterinary Medicine, University of Tennessee, Knoxville, USA.

\section{Received: 27 December 2016 Accepted: 14 August 2017} Published online: 22 August 2017

\section{References}

1. Weese JS. Staphylococcal infections. In: Sellon DC, Long M, editors. Equine Infect Dis Internet. Toronto: Elsevier Health Sciences; 2013. p. 664.

2. Devriese LA, Nzuambe D, Godard C. Identification and characteristics of staphylococci isolated from lesions and normal skin of horses. Vet Microbiol. 1985;10:269-77.

3. Busscher JF. Van Duijkeren E, Sloet van Oldruitenborgh-Oosterbaan MM. The prevalence of methicillin-resistant staphylococci in healthy horses in the Netherlands. Vet. Microbiol. 2006;113:131-6.

4. den Eede A, Martens A, Feryn I, Vanderhaeghen W, Lipinska U, Gasthuys F, et al. Low MRSA prevalence in horses at farm level. BMC Vet Res. 2012;8:213.

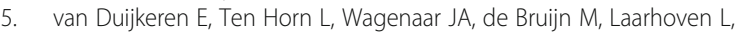
Verstappen K, et al. Suspected horse-to-human transmission of MRSA ST398. Emerg Infect Dis [Internet]. 2011;17:1137-9. Available from: http://www.ncbi. nlm.nih.gov/pmc/articles/PMC3358200/pdf/10-1330_finalL.pdf

6. van Duijkeren E, Moleman M, van Oldruitenborgh-Oosterbaan MM, Multem J, Troelstra A, Fluit AC, et al. Methicillin-resistant Staphylococcus Aureus in horses and horse personnel: an investigation of several outbreaks. Vet Microbiol. 2010; 141:96-102.

7. Weese JS, Caldwell F, Willey BM, Kreiswirth BN, McGeer A, Rousseau J, et al. An outbreak of methicillin-resistant Staphylococcus Aureus skin infections resulting from horse to human transmission in a veterinary hospital. Vet Microbiol. 2006:114:160-4.

8. Weese JS, Archambault M, Willey BM, Hearn P, Kreiswirth BN, Said-Salim B, et al. Methicillin-resistant Staphylococcus Aureus in horses and horse personnel, 2000-2002 141. EmerglnfectDis Centers Dis Control Prev. 2005;11:430-5.

9. Perovic O, Koornhof H, Black V, Moodley I, Duse A, Galpin J. Staphylococcus Aureus bacteraemia at two academic hospitals in Johannesburg. South African Med J. 2006:96:714-7.

10. Magiorakos A-P, Srinivasan A, Carey RB, Carmeli Y, Falagas ME, Giske CG, et al. Multidrug-resistant, extensively drug-resistant and pandrug-resistant bacteria: an international expert proposal for interim standard definitions for acquired resistance. Clin. Microbiol. Infect. [Internet]. Blackwell Publishing Ltd; 2012 [cited 2016 Jun 24];18:268-81 Available from: http://linkinghub. elsevier.com/retrieve/pii/S1198743X14616323

11. Safdar N, Bradley EA. The risk of infection after nasal colonization with Staphylococcus Aureus. Am J Med [Internet]. 2008;121:310-5. Available from: http://www.sciencedirect.com/science/article/pii/S0002934307009746

12. Essa ZI, Connolly C, Essack SY. Staphylococcus aureus from Public Hospitals in Kwa-Zulu-Natal, South Africa-Infection Detection and Strain-Typing. South African J. Epidemiol. Infect. [Internet]. 2009 [cited 2016 Jun 24]; 24:4-7 Available from: http//www.sajei.co za/index.php/SAJEl/article/ viewFile/100/143.
13. Ruzauskas M, Couto N, Siugzdiniene R, Klimiene I, Virgailis M, Vaskeviciute L, et al. Methicillin-resistant coagulase-negative staphylococcus spp. prevalence in Lithuanian dogs: a cross-sectional study. Vet. Arh. 2015:85:175-87.

14. Kern A, Perreten V. Clinical and molecular features of methicillin-resistant, coagulase-negative staphylococci of pets and horses. J Antimicrob Chemother [Internet]. 2013;68:1256-66. Available from: http://jac. oxfordjournals.org/content/68/6/1256.full.pdf

15. Weese JS, Lefebvre SL. Risk factors methicillin-resistant staphylococcus aureus colonization in horses admitted to a veterinary teaching hospital. Can Vet J. 2007:48:921-6.

16. Bagcigil FA, Moodley A, Baptiste KE, Jensen VF, Guardabassi L. Occurrence, species distribution, antimicrobial resistance and clonality of methicillin- and erythromycin-resistant staphylococci in the nasal cavity of domestic animals. Vet Microbiol. 2007;121:307-15.

17. Abrahamsen K. Equine infectious diseases and microbial resistance to antibiotics. Dartmouth Undergradaute J Sci. 2004;1:37-41.

18. Oguttu WJ. Antimicrobial drug resistance of enteric bacteria from broilers fed antimicrobial growth enhancers and exposed poultry abattior workers. Pretoria: University of Pretoria; 2007.

19. Morton TM, Johnston JL, Patterson J, Archer GL. Characterization of a conjugative staphylococcal mupirocin resistance plasmid. DOI Antimicrob. Agents Chemother [Internet]. 1995 [cited 2017 Mar 4];39 Available from: http://aac.asm.org/content/39/6/1272

20. Bjorland J, Steinum T, Sunde M, Waage S, Heir E. Novel plasmid-borne gene qacJ mediates resistance to quaternary ammonium compounds in equine Staphylococcus aureus, Staphylococcus simulans, and Staphylococcus intermedius. Antimicrob. Agents Chemother. [Internet]. 2003 [cited 2017 Mar 4]:47:3046-52 Available from: http://aac.asm.org/cgi/doi/10.1128/AAC. 47.10.3046-3052.2003.

21. Moodley A, Oosthuysen WF, Duse AG, Marais E, South African MSG. Molecular characterization of clinical methicillin-resistant Staphylococcus Aureus isolates in South Africa. J Clin Microbiol [Internet]. 2010;48:4608-11. Available from: http://www.ncbi.n/m.nih.gov/pmc/articles/PMC3008489/pdf/1704-10.pdf

22. Oosthuysen WF, Orth H, Lombard C, Sinha B, Wasserman E. In vitro characterization of representative clinical South African Staphylococcus aureus isolates from various clonal lineages. New microbes new Infect. [Internet]. Elsevier; 2014 [cited 2016 Jun 24];2:115-22. Available from: http:// www.ncbi.nlm.nih.gov/pubmed/25356356.

23. SOUTHAFRICA.COM. Weather of Gauteng | By South Africa Channel [Internet]. South Africa Channels. 2017 [cited 2017 Mar 3] Available from: http://www.southafrica.com/gauteng/climate/.

24. World Weather Online. Weather Averages | Monthly Average High and Low Temperature | Average Precipitation and Rainfall days | World Weather Online [Internet]. 2012 [cited 2017 Mar 3]. Available from: https://www. worldweatheronline.com/johannesburg-weather-averages/gauteng/za.aspx.

25. Quinn PJ, Carter ME, Markey B, Carter GR. Clinical veterinary microbiology. Mosby Wolfe: Edinburgh, Scotland; 1994.

26. Clinical and Laboratory Standards Institute. Performance standards for antimicrobial susceptibility testing; eighteenth informational supplement. CLSI docum. Wayne, PA: Clinicall and Laboratory Standards Institute; 2008.

27. Dohne S, Merle R, Altrock AV, Waldmann KH, Verspohl J, Gruning P, et al. Antibiotic susceptibility of salmonella, campylobacter coli, and campylobacter jejuni isolated from northern German fattening pigs. J Food Prot. 2012;75:1839-45.

28. Royston P. A simple method for evaluating the Shapiro-Francia W' test for non-normality. Underst Stat. 1983;32:297-300.

29. Corporation STATA. Stata MP. Parallel E. 4905 Lakeway drive College Station. TX, USA: Stata Corporation; 2013

30. Leekha S, Diekema DJ, Perencevich EN. Seasonality of staphylococcal infections. Clin Microbiol Infect [Internet]. 2012;18:927-33. Available from: http://onlinelibrary.wiley.com/doi/10.1111/j.1469-0691.2012.03955.x/pdf

31. Fisman DN. Seasonality of Infectious Diseases. https://doi.org/10.1146/ annurev.publhealth.28.021406.144128. Annual Reviews; 2007;

32. Humphreys H, Fitzpatick F, Harvey, Brain J. Gender differences in rates of carriage and bloodstream infection caused by methicillin-resistant Staphylococcus aureus. Are they real, do they matter and why? Clin. Infect. Dis. [Internet]. 2015;61:1708-14. Available from: http://cid.oxfordjournals.org/ content/early/2015/07/21/cid.civ576.full.pdf+html.

33. Schnellmann C, Gerber $V$, Rossano A, Jaquier $V$, Panchaud Y, Doherr MG et al. Presence of New mecA and $\mathrm{mph}(\mathrm{C})$ Variants Conferring Antibiotic 
Resistance in Staphylococcus spp. Isolated from the Skin of Horses before and after Clinic Admission. J. Clin. Microbiol. [Internet]. American Society for Microbiology; 2006 [cited 2016 Jun 24];44:4444-54 Available from: http:// jcm.asm.org/cgi/doi/10.1128/JCM.00868-06

34. Yah SC, Enabulele I, Eghafona NO. The screening of multi-drug resistance (MDR) susceptibilities of Staphylococcus aureus and Staphylococcus epidermidis to methicillin and vancomycin in teaching hospitals in Nigeria. Sudan J. Med. Sci. [Internet]. Omdurman Islamic University; 2008 [cited 2016 Jun 24];2:257-62 Available from: http://www.ajol.info/index.php/sjms/article/ view/38497.

35. Henton MM, Eagar HA, Swan GE, van Vuuren M. Part VI. Antibiotic management and resistance in livestock production. S. Afr. Med. J. [Internet]. 2011 [cited 2017 Jul 15];101:583-6. Available from: http://www. ncbi.nlm.nih.gov/pubmed/21920137.

36. Furr Martin O, Mogg T. Antimicrobial treatment of neonatal foals. Compedium. 2003;25:6.

37. Burton S, Reid-Smith R, McClure JT, Weese JS. Staphylococcus aureus colonization in healthy horses in Atlantic Canada. Can Vet J. 2008;49:797-9.

Submit your next manuscript to BioMed Central and we will help you at every step:

- We accept pre-submission inquiries

- Our selector tool helps you to find the most relevant journal

- We provide round the clock customer support

- Convenient online submission

- Thorough peer review

- Inclusion in PubMed and all major indexing services

- Maximum visibility for your research

Submit your manuscript at www.biomedcentral.com/submit
Biomed Central 